\title{
Aldosterone secretion in patients with septic shock: a prospective study
}

\author{
Secreção de aldosterona em pacientes com \\ choque séptico: estudo prospectivo
}

Rafael Barberena Moraes', Gilberto Friedman', Marina Verçoza Viana', Tiago Tonietto', Henrique Saltz'2, Mauro Antônio Czepielewski ${ }^{3}$

\author{
1 Division of Critical Care Medicine, \\ Department of Internal Medicine. \\ Hospital de Clínicas de Porto \\ Alegre (HCPA), Universidade \\ Federal do Rio Grande do Sul \\ (UFRGS), Porto Alegre, RS, Brazil \\ ${ }^{2}$ Division of Critical Care Medicine, \\ Hospital Nossa Senhora da \\ Conceição, Porto Alegre, RS, Brazil \\ ${ }^{3}$ Division of Endocrinology, \\ Department of Internal \\ Medicine, HCPA, UFRGS, \\ Porto Alegre, RS, Brazil
}

\begin{abstract}
Objective: To assess serum levels of the main factors that regulate the activation of the zona glomerulosa and aldosterone production in patients with septic shock, as well as their response to a high-dose $(250 \mu \mathrm{g})$ adrenocorticotropic hormone (ACTH) stimulation test. Subjects and methods: In 27 patients with septic shock, baseline levels of aldosterone, cortisol, ACTH, renin, sodium, potassium, and lactate were measured, followed by a cortrosyn test. Results: Renin correlated with baseline aldosterone and its variation after cortrosyn stimulation. Baseline cortisol and its variation did not correlate with ACTH. Only three patients had concomitant dysfunction of aldosterone and cortisol secretion. Conclusions: Activation of the zona glomerulosa and zona fasciculata are independent. Aldosterone secretion is dependent on the integrity of the renin-angiotensin-aldosterone system, whereas cortisol secretion does not appear to depend predominantly on the hypothalamic-pituitaryadrenal axis. These results suggest that activation of the adrenal gland in critically ill patients occurs by multiple mechanisms. Arq Bras Endocrinol Metab. 2013;57(8):636-41
\end{abstract}

\section{Keywords}

Aldosterone; hydrocortisone; adrenal insufficiency; septic shock

\section{RESUMO}

Objetivo: Avaliar os níveis séricos dos principais fatores que regulam a ativação da zona glomerulosa e a produção de aldosterona em pacientes com choque séptico, assim como sua resposta ao teste de cortrosina em alta dose $(250 \mu \mathrm{g})$. Sujeitos e métodos: Em 27 portadores de choque séptico, foram aferidos níveis basais de aldosterona, cortisol, ACTH, renina, sódio, potássio e lactato, bem como realizado teste de cortrosina. Resultados: Renina se correlacionou com níveis basais de aldosterona e sua variação após teste de cortrosina. Cortisol basal e sua variação não se correlacionaram com ACTH. Apenas três pacientes apresentaram disfunção concomitante da secreção de aldosterona e cortisol. Conclusões: Ativação das zonas fasciculada e glomerulosa são independentes. Secreção de aldosterona é dependente da integridade do sistema renina-angiotensina-aldosterona, enquanto secreção de cortisol não parece predominantemente dependente do eixo hipotálamo-hipófise-adrenal. Esses resultados sugerem que a ativação da adrenal em pacientes críticos ocorre por múltiplos mecanismos. Arq Bras Endocrinol Metab. 2013;57(8):636-41

\section{Descritores}

Aldosterona; hidrocortisona; insuficiência adrenal; choque séptico

\section{INTRODUCTION}

I n 1936, Hans Selye described the so-called "countershock" response in experimental animals subjected to electric shocks, characterized by adrenal gland swelling, atrophy of the thymus and lymphoid tissues, hyperchloremia and hypernatremia, increased blood volume, and increased diuresis. Selye also demonstrated that adrenalectomized animals were incapable of mounting such a response, thus establishing the asso- ciation between stress and elevated cortisol levels. Selye's work and other studies set the foundation for the routine use of corticosteroid therapy in patients with septic shock, which persisted until the 1990s, when meta-analyses showed that the way in which steroids were used in this setting (late administration of high doses) was not beneficial $(1,2)$. Later knowledge has shown that stressful situations, such as septic shock, activate the hypothalamic-pituitary-adrenal (HPA) axis, conse- 
quently triggering increases in cortisol levels. Patients who are unable to activate this axis exhibit higher mortality rates $(3,4)$. This dysfunction was originally known as relative adrenal insufficiency (RAI) (5), an outdated term that has been often replaced with critical illnessrelated corticosteroid insufficiency (CIRCI) (6). Interest in steroid treatment of patients with septic shock resurfaced in the early 2000s after some trials showed hemodynamic benefits and a possible reduction in mortality with the early use of stress doses of hydrocortisone (200 mg/day) (7-9). Despite the substantial number of publications on the subject, questions remain as to the diagnosis of adrenal dysfunction in critically ill patients.

Most studies in critically ill patients have only assessed the function of the zona fasciculata, the site of glucocorticoid production, even though glucocorticoids are predominantly anti-inflammatory and the main disturbances observed in clinical illness are potentially attributable to mineralocorticoid action (vasopressor unresponsive hypotension, acidosis, and hyperkalemia). Furthermore, the main indications for and benefits of hydrocortisone in septic shock appear to be dependent on its mineralocorticoid effects.

Within this context, a better understanding of the activation mechanism of the zona glomerulosa in critically ill patients is imperative. This layer of the adrenal gland is the site of mineralocorticoid production, particularly aldosterone, which plays several vital roles in the setting of septic shock, such as sodium (and, consequently, fluid) retention and hypertensive effects, both of which are important for the maintenance of tissue perfusion. Some studies have shown that critically ill patients are unable to increase aldosterone output during septic shock, and that this phenomenon occurs secondary to a blunted adrenal response to increased levels of renin, secreted by the juxtaglomerular apparatus. In critically ill patients, this dysfunction is known as hyperreninemic hypoaldosteronism $(\mathrm{HH})(10)$. In other words, critically ill patients would exhibit lower aldosterone levels due to reduced production and/or secretion of aldosterone by the adrenal glands, as occurs with the zona fasciculata and cortisol levels. Insufficient cortisol or aldosterone levels might lead to a major reduction in the activation of mineralocorticoid receptors, consequently leading to circulatory failure.

These aspects highlight the importance of research aimed at assessing the response of aldosterone to a variety of stimuli in critically ill patients. Therefore, this study aimed to assess serum levels of the main factors that regulate the activation of the zona glomerulosa and aldosterone production in patients with septic shock, as well as their response to a high-dose $(250 \mu \mathrm{g})$ adrenocorticotropic hormone (ACTH) stimulation test.

\section{SUBJECTS AND METHODS}

This was a prospective study of 27 adult patients with septic shock admitted to the medical/surgical intensive care unit (ICU) of Hospital de Clínicas de Porto Alegre, a tertiary care referral hospital in southern Brazil. The study was approved by the Research Ethics Committee of the institution and was conducted in accordance with the provisions of the Declaration of Helsinki. Given the severity of the patients' condition, written informed consent was obtained from each patient's closest relative prior to their inclusion in the study.

Patients were eligible for the analysis if they were $\geq$ 18 years of age and had a diagnosis of septic shock according to the criteria of the American College of Chest Physicians/Society of Critical Care Medicine Conference Consensus Committee (11), requiring mechanical ventilation and use of vasoactive drugs despite adequate fluid replacement. Patients were recruited within 96 hours of ICU admission. Exclusion criteria were as follows: history of chronic or acute use of exogenous corticosteroids; use of drugs that might interfere with adrenal function, such as etomidate, angiotensin-converting enzyme inhibitors, angiotensin II receptor antagonists, spironolactone, oral contraceptives, or antifungal agents; acquired immunodeficiency syndrome; history of adrenal insufficiency; HPA axis dysfunction; and pregnancy.

Baseline laboratory studies included blood sample collection for measurement of serum ACTH, renin, potassium, sodium, lactate, aldosterone, and total cortisol levels. Immediately after baseline blood collection, patients underwent an ACTH stimulation test, which consisted of an intravenous injection of $250 \mu \mathrm{g}$ tetracosactide (Synacthen ${ }^{\circledR} 0.25 \mathrm{mg} / \mathrm{l} \mathrm{mL}$; Novartis Pharma, Rueil-Malmaison, France), followed by measurement of aldosterone and cortisol levels at 30 and 60 minutes. All blood samples were obtained and drugs were injected through a central venous catheter. The change $(\Delta)$ in serum aldosterone and cortisol levels was determined by subtracting the baseline level from the peak level. Patients were classified as having CIRCI if basal cortisol was $<10 \mu \mathrm{g} / \mathrm{dL}$ or the variation in total cortisol levels $\left(\Delta_{\text {cortisol }}\right)$ after cortrosyn stimulation was $<9 \mu \mathrm{g} / \mathrm{dL}(\mathbf{6})$. Patients were diagnosed with $\mathrm{HH}$ if the aldosterone/ 
plasma renin activity ratio (Aldo/PRA) was $<2$ and plasma renin activity (PRA) was $>2 \mathrm{ng} / \mathrm{mL} / \mathrm{h}(12)$. The decision to start corticosteroid therapy was made by the physician in charge outside the study, with no influence whatsoever from the investigators. Corticosteroids were always administered after the cortrosyn stimulation test and any other relevant therapeutic interventions.

Patients were followed up for 28 days after the cortrosyn stimulation test. Information was collected on age, sex, type of admission (medical or surgical), source of infection, Acute Physiology and Chronic Health Evaluation (APACHE) II score (13), duration of corticosteroid therapy, duration of vasoactive drug therapy, and outcome (death or survival) at 28 days. Time to withdrawal of vasoactive drugs was defined as successful weaning from vasopressor support for at least 24 hours.

ACTH levels were measured by chemiluminescence (Immulite 1000 Analyzer; Diagnostic Products Corp., Los Angeles, CA, USA), as were total serum cortisol levels (Modular E170 Analyzer; Roche Diagnostics, Mannheim, Germany). Aldosterone (reference range, 2.94-16.15 ng/dL) and PRA (reference range, 0.5 $2.64 \mathrm{ng} / \mathrm{mL} / \mathrm{h}$ ) were measured by radioimmunoassay.

\section{Statistical analysis}

Data were analyzed using the Statistical Package for the Social Sciences (SPSS) version 19.0 (SPSS Corp., Chicago, IL, USA). Normally distributed quantitative variables are expressed as mean \pm standard deviation and asymmetrically distributed variables as median and interquartile range. The chi-square test was used for the assessment of categorical variables, and Student's $t$ test for continuous variables. The Spearman rank correlation coefficient $\left(r_{s}\right)$ was used to test for the correlation between continuous variables, because of their nonnormal distribution. Survival analyses were performed using Kaplan-Meier curves. The log-rank test was used to compare survival curves and determine significant differences at different time points. The level of significance was set at $\mathrm{p}<0.05$. To achieve a moderate correlation between variables (correlation coefficient of 0.6 ), a significance level of $5 \%$ and a statistical power of $90 \%$, a minimum sample size of 19 patients was required.

\section{RESULTS}

A total of 27 patients with septic shock were prospectively recruited and included in the study sample. The demographic, clinical and laboratory characteristics of patients are described in table 1. Mean APACHE II score was 25.4 , and $55.6 \%$ of patients died as a result of this condition (Table 1). Seven patients had PRA $<2 \mathrm{ng} / \mathrm{mL} / \mathrm{h}$ and were therefore excluded from the analysis of Aldo/PRA, as aldosterone activity cannot be assessed at such low PRA levels (12). Particularly striking were the high mortality rate and disease severity (as expressed by high APACHE II scores) observed in our sample, which were nonetheless consistent with the Brazilian literature (14). Correlations between the main hormonal variables analyzed are shown in figure 1. A correlation was detected between PRA and baseline aldosterone levels $\left(r_{s}=0.66, p<0.05\right)$ and between PRA and change in aldosterone levels after cortrosyn stimulation $\left(r_{s}=0.42, p<0.05\right)$. There was no correlation between PRA and levels of ACTH $\left(r_{s}=0.065, p>0.05\right)$, potassium $\left(r_{s}=0.065, p>0.05\right)$, or sodium $\left(r_{s}=-0.36\right.$, $\mathrm{p}>0.05)$. Conversely, baseline aldosterone and cortisol levels were correlated $\left(r_{s}=0.45, p<0.05\right)$. The correlation between baseline aldosterone and change in aldosterone levels after stimulation did not reach statistical significance $\left(r_{s}=0.367, p=0.078\right)$. ACTH did not correlate with baseline cortisol $\left(r_{s}=0.07, p>0.05\right)$, change in cortisol levels $\left(r_{s}=-0.36, p>0.05\right)$, or aldosterone $\left(r_{s}\right.$ $=-0.17, \mathrm{p}>0.05)$. Likewise, baseline cortisol did not correlate with change in cortisol levels $\left(r_{s}=-0.057, p>\right.$ $0.05)$. There were no correlations between potassium, sodium, or lactate and baseline aldosterone or change in aldosterone levels after the cortrosyn stimulation test.

Of 20 patients with PRA $>2 \mathrm{ng} / \mathrm{mL} / \mathrm{h}$, eight had HH (40\%), whereas six had CIRCI (30\%). Only three patients (15\%) exhibited both disorders concurrently.

Both aldosterone and cortisol levels increased significantly after cortrosyn stimulation $(\mathrm{p}<0.05)$, with aldosterone reaching peak levels at 60 minutes in 17 patients (63\%), and cortisol, in 25 patients (92.6\%).

Limiting the analysis to non-dialysis patients at the time of hormone measurements did not change the correlation coefficient between the hormonal variables analyzed. Among these patients, there was an inverse relationship between sodium and potassium $\left(r_{s}=-0.79\right.$, $\mathrm{p}<0.05)$. Mean sodium and potassium concentration among dialysis patients was not statistically different from that of non-dialysis patients (sodium, $142 \pm 6.14$ $\mathrm{mmol} / \mathrm{L}$; potassium, $4.01 \pm 0.75 \mathrm{mmol} / \mathrm{L}$ ).

The probability of death at 28 days was $72.7 \%$ for patients with $\mathrm{HH}$ vs. $44.1 \%$ for patients without $\mathrm{HH}$, and $43 \%$ for patients with CIRCI vs. $58 \%$ for patients without CIRCI. These differences were not statistically significant. The survival curves are shown in figure 2 . 
Table 1. Characteristics of patients $(n=27)$

\begin{tabular}{lc}
\hline & Results \\
\hline Age (years) & $60.6 \pm 17.1$ \\
Sex & \\
Male & $14(52 \%)$ \\
Female & $13(48 \%)$ \\
APACHE II score & $25.4 \pm 8.4$ \\
Admission category & \\
Medical & $14(52 \%)$ \\
Surgical & $13(48 \%)$ \\
Source of infection & \\
Respiratory & $10(37 \%)$ \\
Abdominal & $11(41 \%)$ \\
Urinary & $2(7.4 \%)$ \\
Catheter-related & $1(3.6 \%)$ \\
Other & $3(11 \%)$ \\
Blood glucose (mg/dL) & $137 \pm 39.4$ \\
ACTH (pg/mL) & $22.4(15.4-36.4)$ \\
Cortisol, baseline ( $\mu$ g/dL) & $27.9(21.9-38.4)$ \\
Cortisol $\triangle(\mu g / d L)$ & $16.4(9.8-21.3)$ \\
PRA (ng/mL/h) & $5.4(2.16-15.6)$ \\
Aldosterone, baseline (ng/dL) & $16.3(10.7-29.1)$ \\
Aldosterone $\triangle(\mathrm{ng} / \mathrm{dL})$ & $6.9(3.6-16.8)$ \\
Aldosterone/PRA & $2.22(1.2-3.4)$ \\
$\mathrm{K}^{+}$(mmol/L) & $4.2 \pm 0.64$ \\
Na+(mmol/L) & $145 \pm 6.5$ \\
Lactate (mmol/L) & $2.2(1.6-5.3)$ \\
Duration of corticosteroid therapy (days) & $5.0(1.0-9.0)$ \\
Duration of vasoactive drug therapy (days) & $5.0(2.0-6.0)$ \\
\hline Mortality & $15.6 \%)$ \\
\hline
\end{tabular}

Results are expressed as $n(\%)$, mean \pm standard deviation, or median and interquartile range. APACHE: Acute Physiology and Chronic Health Evaluation; ACTH: adrenocorticotropic hormone; $\triangle$ : variation; PRA: plasma renin activity.

\section{DISCUSSION}

This study evaluated adrenal activation in the setting of septic shock in an attempt to contribute to further elucidation of the pathogenesis of adrenal dysfunction in this population by investigating both the zona fasciculata and the zona glomerulosa of the adrenal gland.

In our sample, PRA was the main determinant of serum aldosterone levels (both at baseline and after cortrosyn stimulation) during shock. This finding, along with the lack of correlation between serum potassium, sodium or ACTH and aldosterone levels, suggests that hypotension and hypovolemia, which act as a major stimulus for renin release, are the main drivers of aldosterone secretion in patients with septic shock. There-
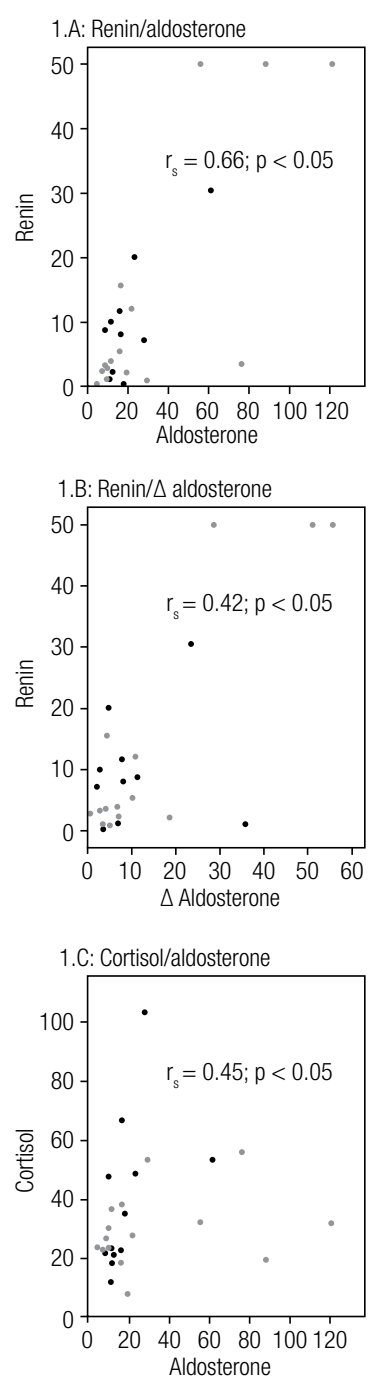

$\Delta$ aldosterone: variation in aldosterone after cortrosyn stimulation

- Survivors . Non-survivors

Figure 1. Correlations between main hormonal variables.

fore, maintenance of the renin-angiotensin system appears to be essential for proper aldosterone production in the critically ill. These findings are consistent with data from a previous study by Cheyron and cols. (15), which was conducted in a population of critically ill patients with cirrhosis.

Lactate, a marker of tissue perfusion and adrenergic status, was not a predictor of PRA in this study. The current literature suggests that, in the zona fasciculata, inflammatory mediators, such as interleukins and tumor necrosis factor, may be more decisive determinants of basal cortisol and increases in cortisol levels (what some researchers term the "adrenal reserve") than ACTH-mediated stimulation by the pituitary gland 


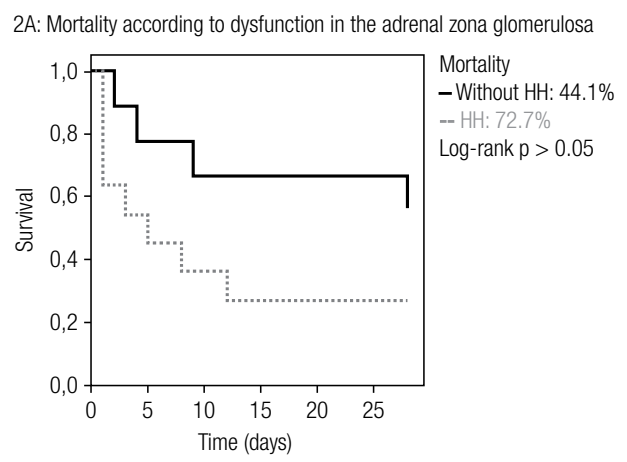

2B: Mortality according to dysfunction in the adrenal zona fasciculate

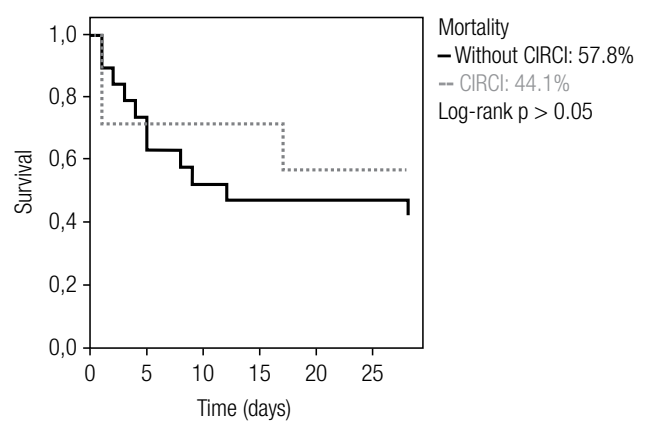

$\mathrm{HH}$ : hyperreninemic hypoaldosteronism;

CIRCl: critical illness-related corticosteroid insufficiency.

Figure 2. Kaplan-Meier survival curves - Analysis of mortality at 28 days according to adrenal dysfunction.

(16). This assertion is, at least in part, supported by our results, which demonstrated no correlation between ACTH and baseline cortisol. Furthermore, in this study, in the setting of septic shock, there was no association between pituitary activity - a determining factor in ACTH release - and activation of the adrenal zona glomerulosa, as evidenced by the lack of association between ACTH and aldosterone levels. Also, there was no correlation between sodium or potassium levels and aldosterone secretion. This finding may be explained by the narrow range of variation in electrolyte levels detected in the sample, which, in turn, may be explained by intensive control of fluid and electrolyte balance in the ICU and also by the fact that $37 \%$ of subjects were on dialysis treatment at the time of hormone measurements. These factors prevented extreme levels of serum sodium and potassium and may, therefore, explain the lack of correlation between these levels and stimulation of the renin-angiotensin-aldosterone system (RAAS). Severe renal impairment may also have contributed to the low renin levels observed in this sample.

Our findings provide in vivo evidence that increases in aldosterone and cortisol secretion result from independent mechanisms. This is consistent with previous studies involving critically ill patients with (17) or without (18) sepsis. Therefore, patients with septic shock may exhibit insufficient glucocorticoid production without concomitant deficiency of mineralocorticoid synthesis, and vice versa. Hypothetically, this dissociation may influence how steroids are replaced in septic patients. Patients with septic shock and mineralocorticoid dysfunction would benefit most from replacement therapy involving agents with mineralocorticoid activity, such as fludrocortisone or hydrocortisone, whereas patients with glucocorticoid dysfunction might benefit most from replacement with predominantly glucocorticoid agents, such as methylprednisolone or dexamethasone. Accurate identification of these dysfunctions, together with determination of $11 \beta$-hydroxysteroid dehydrogenase (11 $\beta$-HSD) type 1 and 2 enzyme activity in order to detect greater sensitivity of glucocorticoid or mineralocorticoid receptors, could contribute to the development of protocols for use of certain steroid agents in specific situations aiming to improve the inflammatory and circulatory responses.

Our findings also give rise to a debate on how patients with these dysfunctions should be classified. The current literature suggests that the term "relative adrenal insufficiency" (RAI) should be replaced with "critical illness-related corticosteroid insufficiency" (CIRCI). However, our findings suggest that RAI and CIRCI should not be used interchangeably, as the zona fasciculate and the zona glomerulosa are activated independently. Dysfunction in aldosterone production at the zona glomerulosa was first identified in the 1980s and termed "hyperreninemic hypoaldosteronism" $(\mathrm{HH})$ in the critically ill (10). Previous studies suggest that $\mathrm{HH}$ may be associated with increased mortality in these patients $(15,19)$. Over the past 20 years, many studies have focused on cortisol production in septic patients, with particular emphasis on the definition of CIRCI and hydrocortisone replacement therapy. However, it is worth noting that the hypertensive effects of hydrocortisone are primarily due to its mineralocorticoid activity. Therefore, the potential benefits of hydrocortisone therapy to septic shock might not be observed in all patients, but perhaps only in those with aldosterone deficiency $(\mathrm{HH})$. This hypothesis has yet to be evaluated in a randomized clinical trial.

In the present study, significant increases in aldosterone and cortisol production occurred after stimulation with $250 \mu$ g cortrosyn, which suggests preservation of adequate adrenal reserve despite the lack of correlation between ACTH and variation in the level of these hormones. This may have been due to high-intensity stimulation by the high-dose cortrosyn test, which is often 
many times greater than that observed during septic shock. Previous studies that found no increases in aldosterone production after an ACTH stimulation test concluded that dysfunction of the zona glomerulosa is not associated with circulating factors, including the RAAS. Unlike Raff and cols. (18), who assessed variation in aldosterone levels only 30 minutes after stimulation, in the present study we assessed variation at 30 and 60 minutes; within this timeframe, $63 \%$ of patients exhibited a peak aldosterone concentration. Therefore, these findings suggest that the RAAS continues to act as a determining factor in the activation of the zona glomerulosa even in the presence of sepsis, as observed in other pathological settings.

A limitation of this study is that we did not interfere with the therapeutic measures prescribed by attending physicians. Therefore, 25 patients $(93 \%)$ received corticosteroids, although they were always administered after measurement of hormone levels. Thus, use of corticosteroid therapy and the small sample size prevented us from assessing clinical outcomes, such as mortality and duration of vasopressor support. Nevertheless, our results are consistent with trials suggesting that $\mathrm{HH}$, detected by Aldo/PRA $<2$, is associated with increased mortality in critically ill patients, representing a potential therapeutic target $(10,12,15,17,19)$. We believe that further prospective trials designed to define whether $\mathrm{HH}$ and its clinical management correlate with improvement in clinical outcomes are still required.

The findings reported herein suggest that activation of the RAAS by hypotension, hypovolemia or other factors is the main determinant of aldosterone secretion in critically ill patients. ACTH, potassium and sodium levels do not appear to play a defining role in the maintenance and production of aldosterone in the adrenal zona glomerulosa in patients with septic shock. Furthermore, this study showed that dysfunction in cortisol production (CIRCI) is not associated with dysfunction in aldosterone production $(\mathrm{HH})$ in the zona glomerulosa. These findings may contribute to the development of new definitions for adrenal dysfunction in critically ill patients. Also, randomized clinical trials involving several different steroids - with predominant glucocorticoid or mineralocorticoid activity - in patients with more clearly defined adrenal disturbances are warranted.

Acknowledgements: the authors declare that there is no conflict of interest that might influence the results and/or discussion reported in this paper. This study was supported by grants from FIPE-HCPA (Fundo de Incentivo à Pesquisa/Hospital de Clínicas de Porto Alegre) and the Graduate Program in Medical Sciences:
Endocrinology, School of Medicine, Universidade Federal do Rio Grande do Sul (UFRGS), Brazil.

\section{REFERENCES}

1. Lefering R, Neugebauer EA. Steroid controversy in sepsis and septic shock: a meta-analysis. Crit Care Med. 1995;23:1294-303.

2. Zeni F, Freeman B, Natanson C. Anti-inflammatory therapies to treat sepsis and septic shock: a reassessment. Crit Care Med. 1997;25:1095-100.

3. Annane D, Sébille V, Troché G. A 3 level prognostic classification in septic shock based on cortisol levels and cortisol response to corticotrophin. JAMA. 2000;283:1038-45.

4. de Jong MFC, Beishuizen A, Spijkstra JJ, Groeneveld AB. Relative adrenal insufficiency as a predictor of disease severity, mortality, and beneficial effects of corticosteroid treatment in septic shock. Crit Care Med. 2007;35:1896-903.

5. Rothwell PM, Udwadia ZF, Lawler PG. Cortisol response to corticotropin and survival in septic shock. Lancet. 1991;337:582-3.

6. Marik PE, Pastores SM, Annane D, Meduri GU, Sprung CL, Arlt $\mathrm{W}$, et al. Recommendations for the diagnosis and management of corticosteroid insufficiency in critically ill adult patients: consensus statements from an international task force by the American College of Critical Care Medicine. Crit Care Med. 2008;36:1937-49.

7. Bollaert PE, Charpentier C, Levy B, Debouverie M, Audibert G, Larcan A. Reversal of late septic shock with supraphysiologic doses of hydrocortisone. Crit Care Med. 1998;6:645-50.

8. Briegel J, Forst H, Haller M, Schelling G, Kilger E, Kuprat G, et al. Stress doses of hydrocortisone reverse hyperdynamic septic shock: a prospective, randomized, double-blind, single-center study. Crit Care Med. 1999;27:723-32.

9. Annane D, Sébille V, Charpentier C, Bollaert BE, François B, Korach $\mathrm{JM}$, et al. Effect of treatment with low doses of hydrocortisone and fludrocortisone on mortality in patients with septic shock. JAMA. 2002;288:862-71.

10. Zipser RD, Davenport MW, Martin KL, Tuck ML, Warner NE, Swinney RR, et al. Hyperreninemic hypoaldosteronism in the critically ill: a new entity. J Clin Endocrinol Metab. 1981;53:867-73.

11. American College of Chest Physicians/Society of Critical Care Medicine Consensus Conference: definitions for sepsis and organ failure and guidelines for the use of innovative therapies in sepsis. Crit Care Med. 1992;20:864-74.

12. Davenport MW, Zipser RD. Association of hypotension with hyperreninemic hypoaldosteronism in the critically ill patient. Arch Intern Med. 1983;143:735-7.

13. Knaus WA, Draper EA, Wagner DP, Zimmerman JE. APACHE II: a severity of disease classification system. Crit Care Med. 1985;13:818-29.

14. Teles JM, Silva E, Westphal G, Filho RC, Machado FR. Surviving sepsis campaign in Brazil. Shock. 2008;30:47-52.

15. du Cheyron D, Bouche Bt, Cauquelin B, Guillotin D, Ramakers D, Daubin $C$, et al. Hyperreninemic hypoaldosteronism syndrome, plasma concentrations of interleukin- 6 and outcome in critically ill patients with liver cirrhosis. Intensive Care Med. 2008;34:116-24.

16. Kwon YS, Suh GY, Jeon K, Park SY, Lim SY, Koh WJ, et al. Serum cytokines and critical illness-related corticosteroid insufficiency. Intensive Care Med. 2010;36:1845-51.

17. du Cheyron D, Lesage A, Daubin C, Ramakers M, Charbonneau P. Hyperreninemic hypoaldosteronism: a possible etiological factor of septic shock-induced acute renal failure. Intensive Care Med. 2003;29:1703-9.

18. RaffH, Findling JW, Diaz SJ, Majmudar MH, WatersVO. Aldosterone control in critically ill patients: ACTH, metoclopramide and atrial natriuretic peptide. Crit Care Med. 1990;18:915- 20.

19. Davenport MW, Zipser RD. Association of hypotension with hyperreninemic hypoaldosteronism in the critically ill patient. Arch Intern Med. 1983;143:735-7. 